\title{
FluConvert and IniFlu: a suite of integrated software to identify novel signatures of emerging influenza viruses with increasing risk
}

Chin-Rur Yang ${ }^{1}$, Chwan-Chuen King ${ }^{2}$, Li-Yu Daisy Liu ${ }^{3,4^{*}}$ and Chia-Chi Ku ${ }^{1 *}$

$\overline{\text { * Correspondence: lyliu@ntu.edu.tw; }}$ chiachiku@ntu.edu.tw

${ }^{3}$ Division of Biometry, Department of Agronomy, NTU, Taipei 10617,

Taiwan, Republic of China ${ }^{1}$ Institute of Immunology, College of Medicine, National Taiwan University (NTU), 1 Jen-Ai Road Section 1, Taipei 10051, Taiwan, Republic of China

Full list of author information is available at the end of the article

\begin{abstract}
Background: The pandemic threat of influenza has attracted great attention worldwide. To assist public health decision-makers, new suites of tools are needed to rapidly process and combine viral information retrieved from public-domain databases for a better risk assessment.

Results: Using our recently developed FluConvert and IniFlu software, we automatically processed and rearranged sequence data by standard viral nomenclature, determined the group-related consensus sequences, and identified group-specific polygenic signatures. The software possesses powerful ability to integrate viral, clinical, and epidemiological data. We demonstrated that both multiple basic amino acids at the cleavage site of the HA gene and also at least 11 more evidence-based viral amino acid substitutions present in global highly pathogenic avian influenza H5N2 viruses during the years 2009-2016 that are associated with viral virulence and human infection.

Conclusions: FluConvert and IniFlu are useful to monitor and assess all subtypes of influenza viruses with pandemic potential. These programs are implemented through command-line and user-friendly graphical interfaces, and identify molecular signatures with virological, epidemiological and clinical significance. FluConvert and IniFlu are available at https://apps.flutures.com or https://github.com/chinrur/ FluConvert_IniFlu
\end{abstract}

Keywords: Highly pathogenic avian influenza viruses, H5N2, Viral and immunological informatics, Risk assessment, Pandemic potential 


\section{Background}

The emergence of novel H5N1 avian influenza virus (AIV) in 1997 resulting in fatalities in humans has raised global concern [1]. As of May 8, 2020, a total of 861 human infections and 455 deaths caused by H5N1 infection had been reported [2]. Thereafter, the re-emergence of highly pathogenic avian influenza (HPAI) A H5Ny subtypes that cause widespread infections in poultry farms and in wild birds since 2003 has greatly attracted public health attention. Interestingly, the H5 AIVs in Asia have evolved faster, having higher viral diversity, greater inter-species transmission, and broader host range than those in Europe and the Americas [3]. Understanding the viral factors which determine the pathogenicity of H5 AIV by timely integration of virological, immunological and epidemiological information will be helpful to establish effective prevention and control measures to minimize future pandemic threats.

The immediate release of the genetic sequences of influenza A viruses combined with collections of tools established for analyzing all types and subtypes of influenza viral sequences have greatly advanced our understanding of the evolution of circulating viruses and their potential risk to animal and human health [4]. Given the fact that multiple mutations across gene segments of influenza viruses can exist and the genomic stability might be influenced by a particular mutation over time [5], new suites of tools are needed to integrate these databases for a better alignment of virological, epidemiological and clinical data in a real-time manner.

Several public-domain databases are available for collecting influenza genetic and epidemiological information. They include: (1) National Center for Biotechnology Information Influenza Virus Database (NCBI-IVD) [6], (2) Global Initiative on Sharing All Influenza Data (GISAID-EpiFlu) [7], and (3) Influenza Research Database (IRD) [8]. While NCBI-IVD provides the complete influenza viral sequences of gene segment across a wide range of years, GISAID-EpiFlu is recognized as a compelling mechanism for rapid sharing of partial or incomplete influenza viral sequences [9]. As for IRD, it contains human and mammalian influenza surveillance data as well as human clinical data associated with viruses, linking host surveillance data to well-characterized virus strains [8].

In this paper, we reported on development of a new suite of integrated software including FluConvert and IniFlu for data processing and analysis. FluConvert provides a series of automated packages to efficiently rearrange genetic data based on standard viral nomenclature [10] and translate the nucleotide sequences into three possible polypeptides from $0,+1$, and +2 open reading frames (ORF) after performing simultaneous multiple sequence alignments. For IniFlu, it is programed to automatically select the correct ORF encoded from corresponding gene segment as well as the spliced isoforms (e.g. NS1, NS2 of NS gene; M1, M2 of M gene). Possible accessory proteins (e.g. PB1N40, PB2-S1, M42) that have been reported in the literatures [11-13] can also be selected by IniFlu. The capability of IniFlu that integrates viral genetic information into clinical and epidemiological surveillance data with high efficiency provides a rapid comparison of variations in viral sequences with epidemiological significance. To this end, we provide the results from analysis of H5N2 HPAI viruses defined by the presence of the hallmark amino acid motif (XRRKRR) at the cleavage site between HA1 and HA2 domains [14]. In addition to these multiple basis amino acid residues in the HA, we demonstrate that several other amino acid substitutions across different gene segments 
of H5N2 avian influenza viruses could be associated with the viral virulence and mammalian infections based on IniFlu-generated polygenic HPAI consensus signature. This suggests that the data analysis platform we report here will be useful to identify novel mutations for risk assessment of AIVs with potential threat to animal and human health.

\section{Methods}

Installation of FluConvert and IniFlu

Both FluConvert and IniFlu are available for free download at https://apps.flutures.com or https://github.com/chinrur/FluConvert_IniFlu. The programs can be automatically installed in a desktop computer after the user perform execution files which is found in downloaded folders. The operating system of the computer requires Microsoft Windows 10 (version 1903 or later version) equipped with Microsoft Office 365 or Office Excel 2016 (or later version, 64-bit) for installation and Java 6 (or later, 64-bit) to perform each software. When open FluConvert, the user will be asked to import data which have been pre-downloaded from NCBI-IVD or GISAID-EpiFlu (Step 1 of Fig. 1)

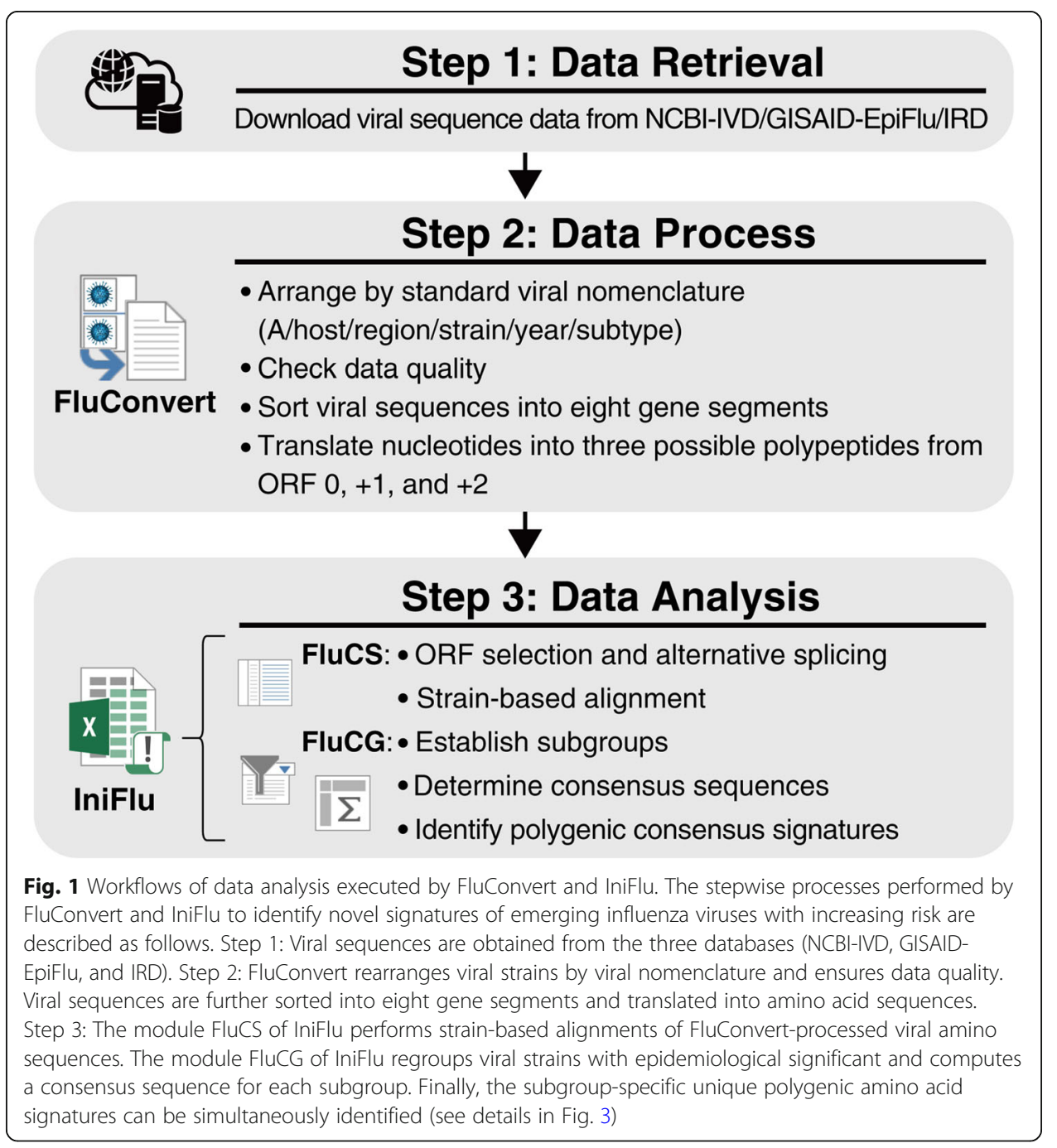


following instructions provided on the website. It will take minutes to hours to complete the process depending on the quantity of data entry. Once FluSeed Dataset has been processed by FluConvert (Step 2 of Fig. 1), IAV sequences can be analyzed by IniFlu (Step 3 of Fig. 1). We also include a detail step-by-step user's guide in Readme which can be found at https://apps.flutures.com website.

\section{FluConvert: a tool to process downloaded sequences}

FluConvert automatically processes downloaded sequence files (".FASTA) using the command-line interface (CLI) by batch (shell) scripts operated in a Microsoft Windows environment. It consequently performs (1) name and quality checking for downloaded sequences, (2) separation of sequences into eight gene segments, (3) multiple alignment of DNA sequences within clusters, (4) translation of DNA sequences into three possible polypeptides from ORF $0,+1$, and +2 , and (5) multiple alignment of amino acid sequences within clusters. The functions of FluConvert are to unify the arrangement of genetic information and then to convert nucleotide sequences of cDNA to amino acid sequences for multiple alignment at the protein level.

\section{Arrangement and quality checking for downloaded sequences}

All sequences downloaded from the NCBI-IVD and the GISAID-EpiFlu databases are rearranged according to the standard influenza viral nomenclature in the order of type, host, region, strain, year, and subtype within the parentheses [10]. Secondly, rearranged sequences are inspected, and the gene segments are deleted when they met any conditions in the "excluding list" generated for quality checking. Entries retrieved from NCBI-IVD and GISAID-EpiFlu databases were deleted according to "excluding list" to remove duplicates, incomplete sequences, or those with error information. Downloaded entries that are later saved to FluSeed Dataset have never been modified or corrected for any purposes. This is to ensure that the information remains original and the features of genetic sequences are kept unaltered during FluConvert processing. The three major error conditions of viral sequence information are: (1) lacking complete viral nomenclature, having mixed subtypes, belonging to lab strains or showing errata in public database records, (2) finding duplicate sequence records in any of the public databases, and (3) sequences longer than the expected lengths for different segments (e.g. PB1 > 2500 bp, PB2 > 2500 bp, PA > 2400 bp, HA > 1900 bp, NP > 1700 bp, NA > 1600 bp, M > $1150 \mathrm{bp}$, and NS $>1050 \mathrm{bp}$ ), or having redundant sequences or those containing more than 60 unknown nucleotides (denoted as ' $n$ '). Finally, all the sequences that had passed the excluding list's quality check without entering the excluding list were used to create a new dataset called the "FluSeed Dataset" and subjected to IniFlu analysis.

As noted, entries retrieved from these public domain databases have never been modified or corrected after downloading. This ensures to keep information original and features of genetic sequences are not lost during FluConvert processing. Moreover, FluSeed Dataset is used for IniFlu analysis and has never been intended to make publicly accessible.

\section{Multiple sequence alignment and amino acid translation}

The genome of influenza A virus contains eight RNA segments. Therefore, FluConvert first divides the genetic sequences in FluSeed Database into eight clusters by the MAFF 
$\mathrm{T}$ multiple sequence alignment program (version 7.429) with fast Fourier transform [15]. All sequences in each of the eight gene segments are then translated into three possible polypeptides from ORF $0,+1$, and +2 by EMBOSS Transeq (version 6.5) [16]. Nucleotide sequences and amino acid sequences in each of the eight gene segments in the FluSeed Database are subject to multiple alignment by MAFFT again with different optimizing parameters based on sequence lengths and the numbers of viral strains or files [i.e. L-INS-i (accurate) for alignment of < 200 viral strains/files; FFT-NS-2 (fast) for alignment of $<\sim 30,000$ viral strains/files to obtain maximal efficiency; and PartTree (fast) for alignment of $>\sim 30,000$ viral strains/files] [17]. Results of sequence alignments from the same ORF were saved as comma-delimited (csv) text files.

IniFlu: a viral information viewer and analyzer

IniFlu, a Visual Basic Application (VBA) program for Microsoft Office Excel 2019 worksheet, has a user-friendly graphical interface (GUI) to combine viral information, amino-acid sequences and epidemiological data for further analyses. IniFlu has two modules, "FluCS" (which stands for "Flu Cross-Segment alignment") and "FluCG" (which stands for "Flu Comparative Grouping"). FluCS matches the aligned sequences according to the standard viral nomenclature of the strains after encoding protein from ORF selection and alternative splicing. FluCG visualizes different epidemiologically specific (such as time-, area-, host-, age-, gender-specific) consensus signatures obtained (shown in Fig. 4), providing not only the clinical information of the viral sequences but also their epidemiological characteristics.

\section{FluCS: strain-based amino acid sequence alignment}

FluCS groups the amino-acid sequences of each gene segment according to FluSeed Dataset (Fig. 2a). FluCS is also programed to automatically select the correct ORF of each viral protein as well as the alternatively spliced isoforms. Accessory proteins, e.g. PB1-N40, PB2-S1 and M42 [11-13] can be assigned to the viral segment group of PB1, $\mathrm{PB} 2$, and $\mathrm{M}$, respectively. PB1-F2 which is translated by a second ORF in the +1 frame $[11,18]$ is selected and assigned to an independent group. As a result, a total of 11 viral segment groups (PB1, PB2, PA, HA, NA, NP, M1, M2, NS1, NS2, and PB1-F2) is established for strain-based alignment (Fig. 2b, c). The position of each residue is numbered based on the first methionine residue of that gene segment determined by FluCS (e.g. HA of H5N2 subtype is numbered by H5 numbering system) [19].

\section{FluCG: comparative sequence analysis}

FluCG chooses the most representative amino acid at each residue of a particular gene segment by computing the most frequent amino acid among all strains within the studied subgroup. If there are more than two amino acids occurring at the same frequency, one is chosen by alphabetic order. Residues that appear at stop codons or deleted codons are marked. Through this process, the consensus sequence can be created for a particular subgroup [20]. The unique residues in each subgroup can also be identified by aligning two consensus sequences and are called "consensus signatures". All the unique amino acids appearing at each residue of the consensus signature are thoroughly examined and compared to verify the unique amino acid is present only in the 


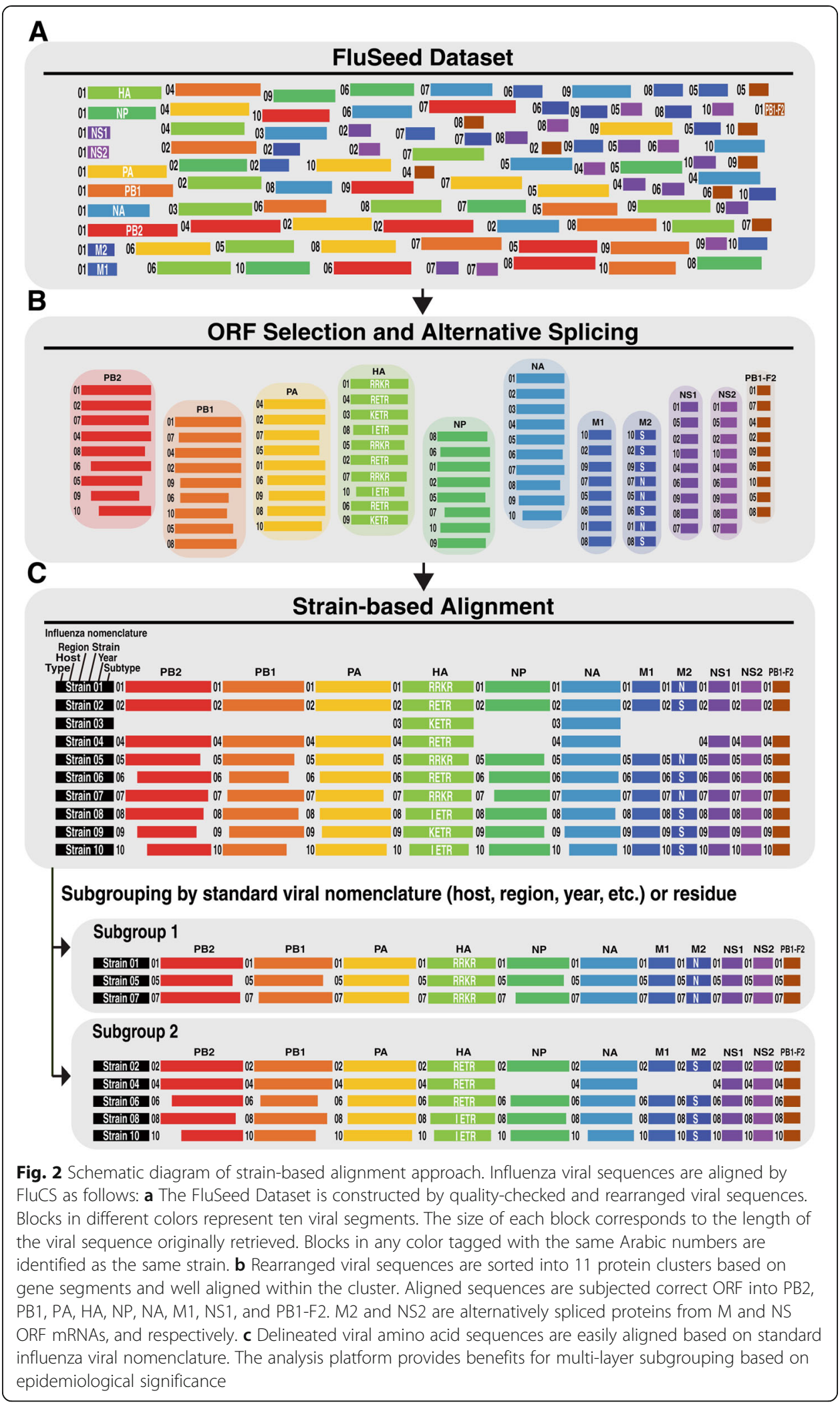


particular subgroup. Finally, all possible 20 amino acids, stop codons, and deletions are all examined and presented in a substitution table (as Fig. 5).

\section{Results}

Influenza viral sequences and data processing

The genetic sequences and epidemiological information of influenza viruses in one public domain database are not properly linked to the other. To maximize the information coverage for a particular AIV subtype for further analysis, we have developed the FluConvert program to combine all data available from these databases and automatically process them in one format. Viral sequences that had passed quality check after excluding incomplete or erroneous ones to ensure correct genetic information are used for constituting the FluSeed Dataset. Sequences in the FluSeed are subsequently rearranged to standard nomenclature in the order of influenza virus of type/host/region/ strain/year (HxNy subtype) and segregated into eight gene segments. Amino acid sequences are translated from nucleotide sequences for alignment (Fig. 1).

\section{Viral strain-based sequence alignment}

Continuous mutations in HPAI A (H5) viruses have been attributed to outbreaks at poultry farms and sporadic human infections [21]. Since mutations can occur across several gene segments in the genome of AIVs, multiple alignments for all viral strains based on the subgroup of interest (i.e. host, region, year, a particular residue, etc.) rather than by gene segment (i.e. HA, NA, PB2, etc.) will be useful to identify multiple amino acid types associated with viral pathogenicity in animals or the potential risk for human infections. To achieve the goal, we have developed the IniFlu platform to integrate the processed viral sequences, clinical and epidemiological information into the FluSeed Dataset. IniFlu can function to present all the information imported from FluSeed in worksheet outputs for visual crosssegment examinations simultaneously. Once all of the viral strain information is correctly arranged by FluCS, strain-based alignment can be quickly performed as illustrated in Fig. 2 .

\section{Identification of polygenic consensus signatures}

Genetic evolution of zoonotic influenza viruses is a polygenic trait. Amino acid substitutions or mutations at species-associated signature positions may increase viral pathogenicity or mammalian adaptation in a broader host range [22]. Since such mutations are not limited to one gene and can simultaneously occur in multiple gene segments, identification of the polygenic consensus signatures for a particular subgroup of viral strains offers an opportunity to monitor the changing landscape of AIVs over time with epidemiological significance. The module FluCG of IniFlu can quickly group viral strains into different subgroups and deduce the consensus sequence of each subgroup by computing and determining the most representative (i.e. most frequent) amino acid at each position of the whole genome, which can differentiate between the compared subgroups. All unique amino acid residues represented in the subgroup constitute the polygenic consensus signature (Fig. 3).

\section{Polygenic consensus signatures of the HPAI H5N2 viruses}

Duplicate entries of downloaded influenza viral genetic sequences could possibly occur when (1) the entry was submitted to both NCBI-IVD and GISAID-EpiFlu databases, (2) 


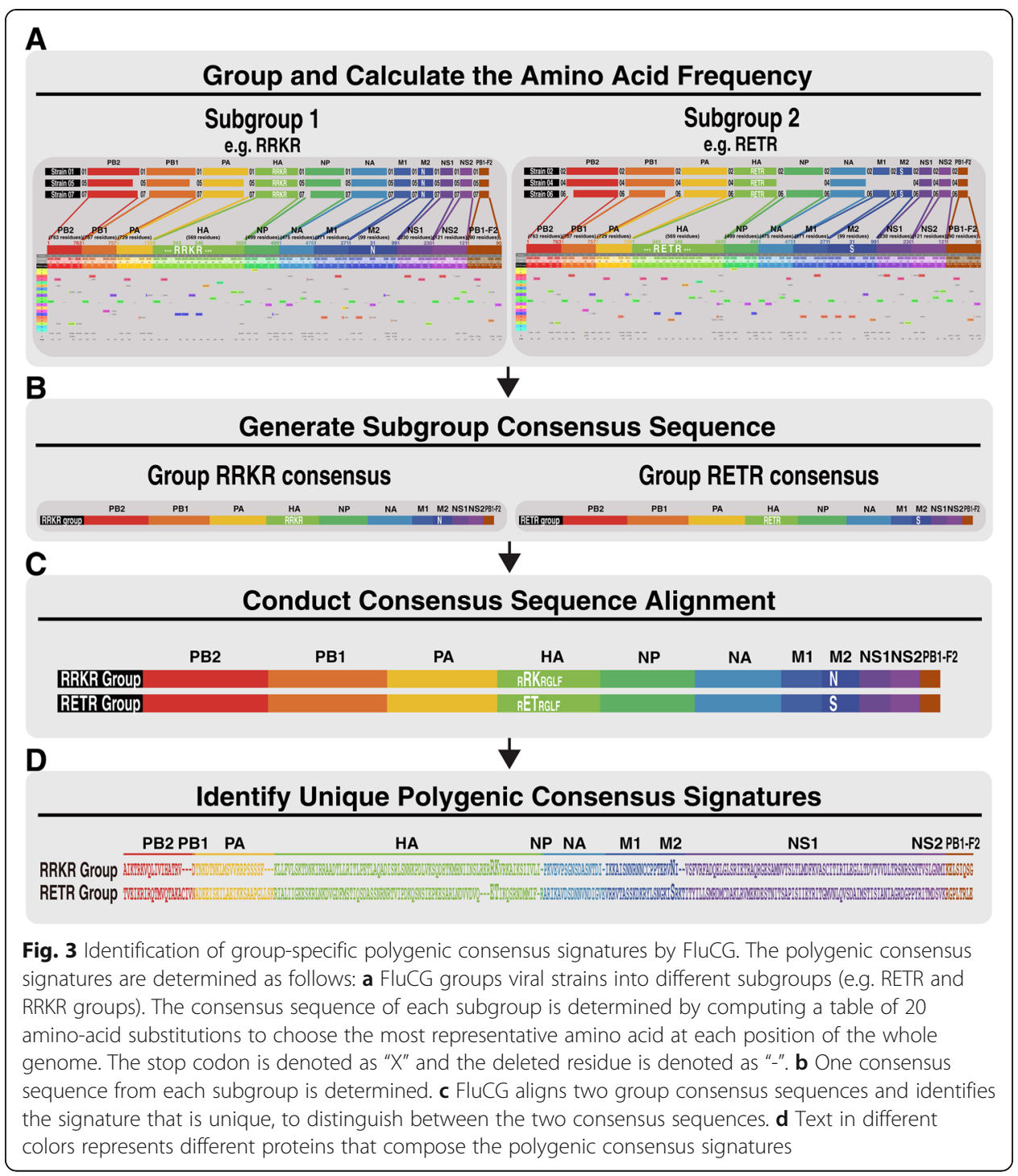

the entry was submitted to one database more than once, or (3) data entries imported from NCBI-IVD co-existed in GISAID-EpiFlu database. To obtain the accurate count of $\mathrm{H} 5 \mathrm{~N} 2$ virus strains downloaded from different public domain databases, FluConvert is programmed to automatically remove duplicate entries. Only one copy of the gene segment of a single virus strain is kept in FluSeed Dataset.

As of July 1, 2017, the H5N2 FluSeed Dataset was comprised of a total of 6746 $(6443+303=6746)$ unique gene segments that belong to $1151(1099+52=1151)$ H5N2 viruses. Amongst which, 6443 gene segments of 1099 H5N2 strains were downloaded from NCBI-IVD and 303 segments of 52 H5N2 strains were downloaded from GISAID-EpiFlu, respectively. Qualified genetic sequences were rearranged by FluConvert to unify the nomenclature format. Corresponding epidemiological information and clinical data for each strain were integrated through the IniFlu platform. Since several studies have demonstrated that the presence of multiple basic amino acids at the cleavage site between HA1 and HA2 junctional sequence is a hallmark for increasing viral pathogenicity and virulence in the avian host and humans [14], we compared the 
molecular signature in the H5N2 viral strains with (RRKR group) or without (RETR group) polybasic residues at the cleavage site in the HA gene. The earliest record of H5N2 viruses was reported in 1972 and all of the 470 strains isolated during 19722008 appeared to have RETR sequence motif at the HA cleavage site. H5N2 viruses with the RRKR sequence motif in the HA only appeared after year 2009. To avoid bias towards evolutionary perspective, we excluded $\mathrm{H} 5 \mathrm{~N} 2$ viruses that were isolated before 2008 and only kept the H5N2 viruses isolated from year 2009 to 2016 in the H5N2 FluSeed Dataset for consensus signature analysis of both groups. As a result, there were 165 strains of H5N2 viruses with RETR marker and 138 strains with RRKR marker.

The consensus sequence analysis by FluCG identified 247 unique amino acid residues differentially presented between RRKR and RETR groups in the whole genome of H5N2 AIVs. Since these unique residues were present across several viral segments, we wanted to know which gene segment might present the most unique residues that may distinguish H5N2 viruses with the REKR marker from those RRKR. Table 1 shows the frequencies of the characteristic substitutions that occurred at a particular gene segment. We found that NS1 had the highest substitutions $(N=69,30 \%)$, followed by HA $(N=77,13.53 \%)$, and PB1-F2 $(N=8,8.89 \%)$. There were much less substitutions in NP $(N=1,0.2 \%)$ and PB1 $(\mathrm{N}=1,0.13 \%)$ of $\mathrm{H} 5 \mathrm{~N} 2$ viruses (Table 1$)$.

To investigate what substitution at a particular residue or residues could be associated with the RRKR phenotype, the polygenic consensus signatures determined from the constellation of the 247 distinct residues as described in Table 1 were further analyzed (Fig. 4). In search of information on amino acid substitutions in the influenza viruses that are associated with increased viral virulence or drug resistance [23] reported in the public domain database IRD-SFVT (Sequence Feature Variant Types) by IniFlu analysis, we found that substitutions in HA, including T124I, D142E, E228K, P233S, V336S in HA, G631S in PA that are related to increasing pathogenicity [24-26] were present in the RRKR signature. Other variations in the HA of the RRKR signature involved in the increase of $\alpha-2.6$ receptor binding in mammalian cells such as S139P, S145L, S149A, and I226V [27-30] were also found in our analysis. Notably, the fact that IniFlu identified the substitution of $\mathrm{S} 31 \mathrm{~N}$ in the M2 of the RRKR signature suggests that H5N2 HPAI may have a decreased sensitivity to amantadine and rimantadine [31] (Fig. 4). All of the unique 11 consensus signatures were re-examined and verified from FluCG-generated substitution table (Fig. 5). Taken together, IniFlu can identify additional substitutions across the gene segments of H5N2 that are highly associated with viral pathogenicity and/or antiviral drug resistance.

Table 1 The 247 residues differentially occurring between RRKR and RETR consensus signatures are polygenic

\begin{tabular}{lllllllllllll}
\hline & \multicolumn{1}{l}{ Influenza viral proteins } & 11 & & & \\
\cline { 2 - 11 } & PB2 & PB1 & PA & HA & NP & NA & M1 & M2 & NS1 & NS2 & PB1-F2 \\
\hline Segment size $^{\mathbf{a}}$ & 763 & 757 & 729 & 569 & 499 & 475 & 271 & 99 & 230 & 121 & 90 \\
No. of consensus signatures & 20 & 1 & 23 & 77 & 1 & 18 & 15 & 8 & 69 & 7 & 8 \\
between groups $^{\mathbf{b}}$ (\%) & $(2.62)$ & $(0.13)$ & $(3.16)$ & $(13.53)$ & $(0.2)$ & $(3.79)$ & $(5.54)$ & $(8.08)$ & $(30)$ & $(5.79)$ & $(8.89)$ \\
\hline
\end{tabular}

${ }^{a}$ : The H5N2 viral genome is composed of 4603 amino acid residues divided into 11 viral proteins. The size of each segment is indicated by the number of residues as shown

${ }^{b}$ : Unique amino acid residues are identified by comparing the consensus sequences between the two studied subgroups. Numbers shown are the counts of the characteristic residues in each viral protein. The variations in each viral protein are expressed by the percentage of unique residues indicated in the parentheses 


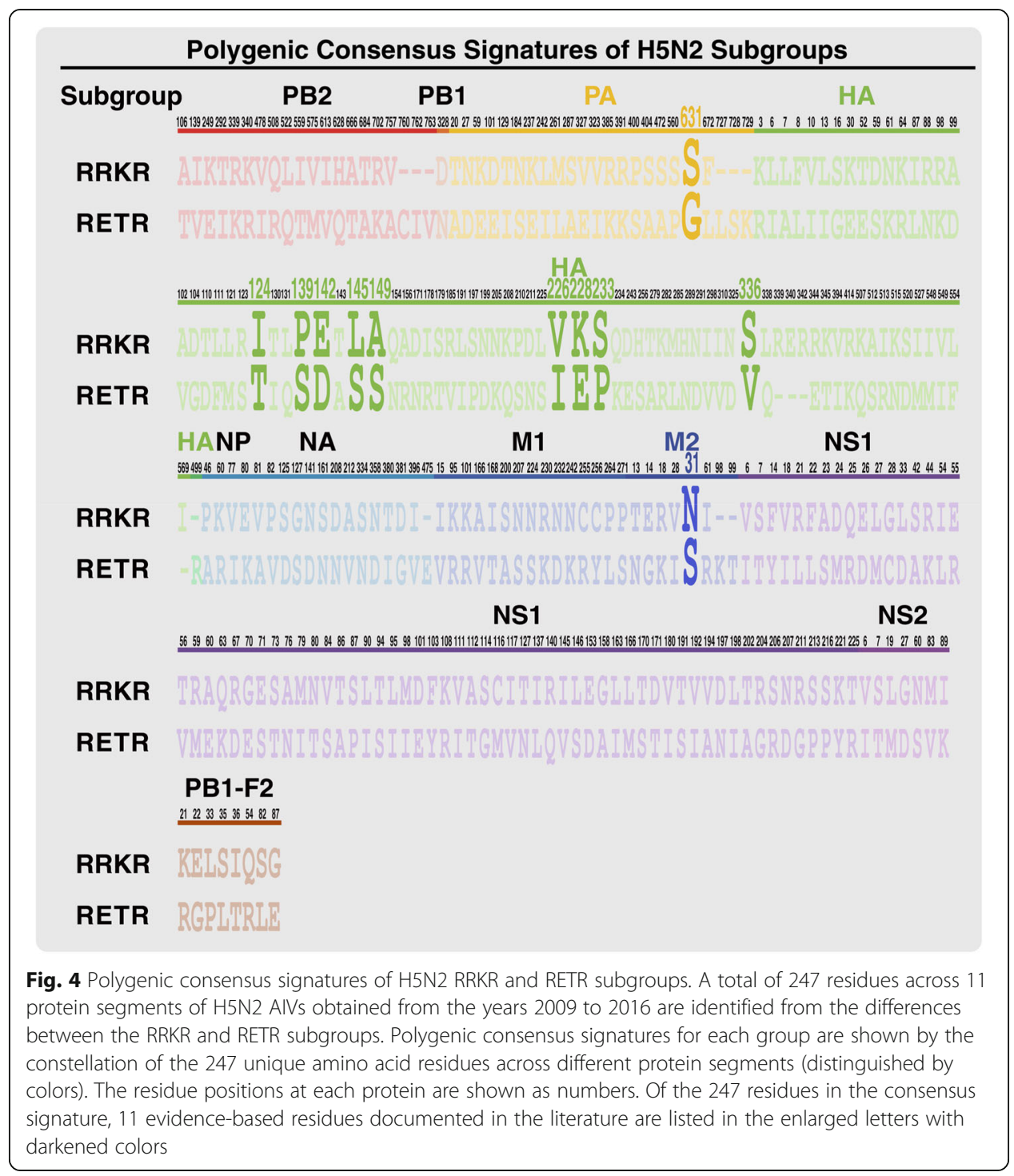

\section{Discussion}

Influenza is an important disease in humans and animals. The 13,588-base-pair RNA genome segregated into eight gene segments continues to mutate randomly at $2 \times 10^{-6}$ mutations per site per infectious cycle [32]. The high activity in the reassortment of segmented influenza viral genes derived from different host species has posed a great threat to public health. Numerous tools have been developed to analyze influenza genetic sequences to monitor the changes and evolution of these viruses over time in nature. In this study, we have added two integrated analysis tools, FluConvert and IniFlu, to the endeavor.

Several analysis tools for IAV genetic sequences are available online to determine antigenic characteristics of IAVs based on the genomic sequences of a particular gene segment and associated epidemiological information. Here we compare a recently published program FluPhenotype [33] with IniFlu. FluPhenotype is a web-based tool. Briefly, IAVs amino acid markers associated with human adaptation, enhanced virulence, and drug resistance, etc. that have been reported in the literatures are captured to the Data list of FluPhenotype. The input genetic sequences of IAVs are mapped with the list and the 


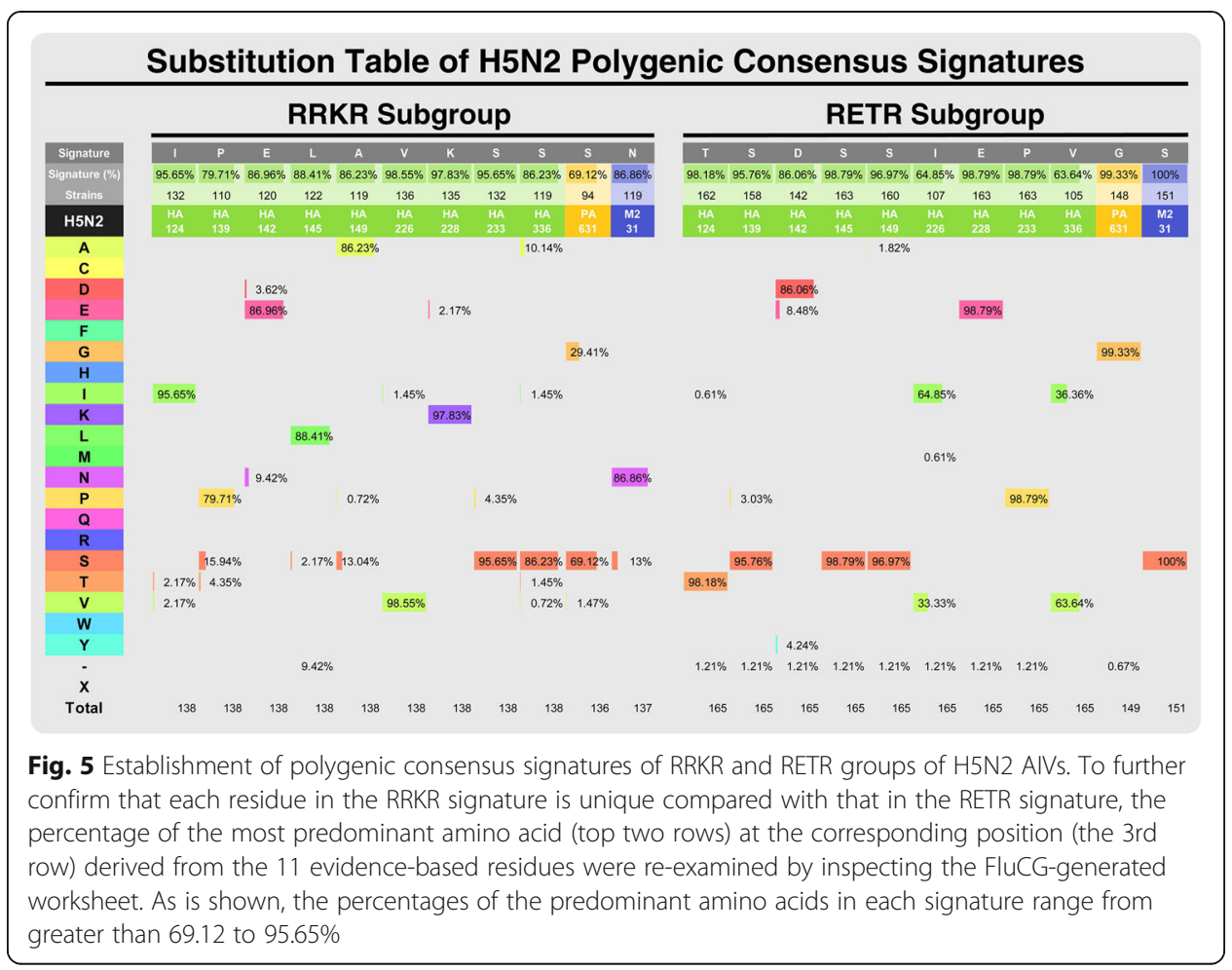

antigenic characteristics of the IAVs of interest are rapidly determined. FluPhenotype also has the capacity to predict IAV HA subtype and viral hosts based on the input genomic or protein sequences [33]. Although the Data list used in FluPhenotype is reportedly updated every half a year, any newly identified or undefined molecular markers that have not been made available in the literature would not be captured and mapped in a timely manner [33].

In comparison with FluPhenotype, FluConvert is used to sort IAV genetic entries that are downloaded from different public domain databases into eight gene segments based on the name of the gene segment (e.g. PB2, PB1, PA, ... etc.). FluConvert subsequently rearranges the information tagged to each entry according to the standard IAV nomenclature in the order of type, host, region, strain, year, and subtype, thereby assigning a unique name to each virus. Therefore, gene segments that have the same name will be grouped as one strain. The capability of FluConvert that determines the correct protein sequences encoded by each viral gene segment and their spliced isoforms as well as accessory proteins results in 11 viral protein clusters in the FluSeed Dataset for strain-based alignment by FluCS.

Since FluCS can align a larger number of viral strains at one time, it saves time on cross-referring of each genetic sequence in NCBI-IVD/GISAID-EpiFlu by accession number. Additionally, the ability of FluConvert to combine information between databases can collect all available influenza genetic data as much as possible by avoiding the exclusion from incomplete information in the depository database. Data in the FluSeed Dataset can be maintained up to date by downloading newly depository of influenza viral genetic data in public domain databases by users.

There are two advantages of IniFlu-performed strain-based alignment and consensus sequence analysis. First, genetic sequences of a viral strain lacking eight complete gene segments can be compared and included for consensus sequence analysis. Second, once 
the information is properly aligned, sequence data can be easily re-grouped for a generating group-specific consensus sequence. As a result, polygenic consensus signatures composed of unique molecular positions across all gene segments that are associated with a particular phenotype will be determined. As demonstrated from the analysis of the sample H5N2 FluSeed Dataset by comparing the group-specific polygenic consensus signatures between the RRKR and the RETR groups, we identified that at least 247 positions of the total 303 H5N2 AIV strains from 2009 to 2016 were able to differentiate these two groups, and 11 of these substitutions have been experimentally demonstrated for the significance in crossing over between host species (e.g. S139P, S145L, S149A, and I226V in HA) [27-30], antiviral drug amantadine and rimantadine resistance (S31N in M2) [31] or increasing viral pathogenesis (e.g. T124I, D142E, E228K, P233S, and V336S in HA, and G631S in PA) [24-26]. Although there have not been reports of fatal human cases of H5N2, human infection of this AIV subtype have occurred, as documented in seroepidemiological studies [34, 35]. These substitutions together with those residues involved in enhancing receptor binding to mammalian cells [14] have suggested the potential threat to human health caused by H5N2 AIV strains with an RRKR phenotype.

Taken together, we reported the newly developed analysis tools FluConvert and IniFlu, which exhibit high capacity and efficiency in data processing, analyzing, and combining large amounts of the most comprehensive influenza viral information retrieved from different public domain databases without making any modifications on downloaded genetic information. These tools not only provide a versatile and rapid platform for real-time analysis to determine consensus sequences, but also identify molecular markers with high pathogenicity in chickens as well as with interspecies transmission to humans. FluConvert and IniFlu are particularly useful in risk assessment by monitoring and analyzing the increasing trends of important amino acids of many animal influenza viruses with pandemic potential. While IniFlu is first designed for type A influenza viruses, the software can easily adapt to investigate other emerging viruses with appropriate modifications on the worksheet template. The software reported in this study provides a useful tool for rapidly identifying molecular signatures with virological, epidemiological and clinical significance.

\section{Conclusions}

The rapid evolution of H5 AIVs in Asia has increased the threat in agricultural safety and human health. The timely monitoring in the changes of AIV that have increasing risk are important for public health-policy makers. FluConvert and IniFlu reported in this study are demonstrated for their efficiency in combining and analyzing virological, epidemiological and clinical information from different public domain databases. Finally, identification of polygenic signature for AIVs with high risk instead of variations at one single gene segment of influenza viruses will be beneficial to assist a better risk assessment to prevent pandemic influenza.

\section{Availability and requirements}

Project name: FluConvert_IniFlu

Project home page: https://apps.flutures.com or https://github.com/chinrur/FluConvert_IniFlu 
Operating system(s): Microsoft Windows 10 or later version (64-bit)

Programming language: Batch (shell) scripts and VBA 7.1

Other requirements: Microsoft Office Excel 365 or Excel 2016 or later version (64-

bit); Java 6 or higher version

License: MIT License.

Any restrictions to use by non-academics: No restrictions on use by nonacademics.

\section{Abbreviations}

AIV: avian influenza virus; CLI: command-line interface; EMBOSS: The European Molecular Biology Open Software Suite; FluCG: Flu Comparative Grouping; FluCS: Flu Cross-Segment alignment; GISAID: Global Initiative on Sharing All Influenza Data; GUI: graphical interface; HPAl: highly pathogenic avian influenza; IAV: influenza A virus; IRD: Influenza Research Database; IVD: Influenza Virus Database; MAFFT: multiple sequence alignment program with fast Fourier transform; NCBI: National Center for Biotechnology Information; ORF: open-reading-frame; VBA: Visual Basic Application; WHO: World Health Organization

\section{Acknowledgements}

We express our special thanks to Dr. Hsiang-Hsuan Sung at the Institute of Microbiology and Immunology, College of Medicine, National Defense Medical Center (NDMC), Taiwan for his insights in initiating the project.

\section{Authors' contributions}

CRY created the software, carried out the analysis and drafted the manuscript; CCK designed epidemiological study; LYL validated the software and substantially revised the writing in Methods; CCK interpreted the data and was a major contributor in writing the manuscript. All authors read and approved the manuscript.

\section{Funding}

CRY was the recipient of the fellowship from the Sustainability Science Research Program, Academia Sinica, Taiwan (grant no. AS-SS-106-04-4). Grants from the Ministry of Science and Technology, Taiwan (MOST 107-2320-B-002008;108-2320-B-002-038) supported research-related logistic expenses and publication fees. None of the funding body plays any role in the design of the study and collection, analysis, and interpretation of data and in writing the manuscript.

\section{Availability of data and materials}

The H5N2 Dataset generated and analyzed during the current study are available in the NCBI-IVD (https://www.ncbi. nIm.nih.gov/genomes/FLU/) and GISAID-EpiFlu databases (https://www.gisaid.org/). These analysis tools are not intended for use in public domain but only for processing data retrieved from public databases. Their use would not breach the data access agreement of GISAID. Abiding by GISAID-EpiFlu Database Access Agreement, these tools will not generate new database for public access or make any annotation, correction, or modification of data submitted to GIASID EpiFlu Database.

\section{Ethics approval and consent to participate}

Not applicable.

\section{Consent for publication}

Not applicable.

\section{Competing interests}

The authors declare that they have no competing interests.

\section{Author details}

${ }^{1}$ Institute of Immunology, College of Medicine, National Taiwan University (NTU), 1 Jen-Ai Road Section 1, Taipei 10051, Taiwan, Republic of China. ${ }^{2}$ Institute of Epidemiology and Preventive Medicine, College of Public Health, NTU, Taipei 10055, Taiwan, Republic of China. ${ }^{3}$ Division of Biometry, Department of Agronomy, NTU, Taipei 10617, Taiwan, Republic of China. ${ }^{4}$ Department of Agronomy, National Taiwan University, No. 1, Section 4, Roosevelt Rd, Taipei 10617, Taiwan.

Received: 10 April 2020 Accepted: 9 July 2020

Published online: 18 July 2020

\section{References}

1. Chan PKS. Outbreak of Avian Influenza A(H5N1) Virus Infection in Hong Kong in 1997. Clin Infectious Diseases. 2002; 34(Supplement_2):S58-64.

2. Cumulative number of confirmed human cases of avian influenza $A(H 5 N 1)$ reported to WHO [https://www.who.int/ influenza/human_animal_interface/H5N1_cumulative_table_archives/en/]. Accessed 8 May 2020.

3. Neumann G, Chen H, Gao GF, Shu Y, Kawaoka Y. H5N1 influenza viruses: outbreaks and biological properties. Cell Res. 2010;20(1):51-61.

4. Hadfield J, Megill C, Bell SM, Huddleston J, Potter B, Callender C, Sagulenko P, Bedford T, Neher RA. Nextstrain: real-time tracking of pathogen evolution. Bioinformatics. 2018;34(23):4121-3. 
5. Arai Y, Kawashita N, Hotta K, Hoang PVM, Nguyen HLK, Nguyen TC, Vuong CD, Le TT, Le MTQ, Soda K, et al. Multiple polymerase gene mutations for human adaptation occurring in Asian H5N1 influenza virus clinical isolates. Sci Rep. 2018;8(1):13066.

6. Bao Y, Bolotov P, Dernovoy D, Kiryutin B, Zaslavsky L, Tatusova T, Ostell J, Lipman D. The influenza virus resource at the National Center for biotechnology information. J Virol. 2008;82(2):596-601.

7. Shu Y, McCauley J. GISAID: Global initiative on sharing all influenza data - from vision to reality. Euro Surveill. 2017; 22(13):30494.

8. Zhang Y, Aevermann BD, Anderson TK, Burke DF, Dauphin G, Gu Z, He S, Kumar S, Larsen CN, Lee A, et al. Influenza research database: an integrated bioinformatics resource for influenza virus research. Nucleic Acids Res. 2016;45(D1):D466-74.

9. Elbe S, Buckland-Merrett G. Data, disease and diplomacy: GISAID's innovative contribution to global health. Global Chall. 2017;1(1):33-46.

10. WHO. A revision of the system of nomenclature for influenza viruses: a WHO Memorandum. Bull World Health Organization. 1980;58(4):585-91.

11. Wise HM, Foeglein A, Sun J, Dalton RM, Patel S, Howard W, Anderson EC, Barclay WS, Digard P. A complicated message: identification of a novel PB1-related protein translated from influenza a virus segment 2 mRNA. J Virol. 2009;83(16):8021-31.

12. Yamayoshi S, Watanabe M, Goto H, Kawaoka Y. Identification of a novel viral protein expressed from the PB2 segment of influenza a virus. J Virol. 2016:90(1):444-56.

13. Wise HM, Hutchinson EC, Jagger BW, Stuart AD, Kang ZH, Robb N, Schwartzman LM, Kash JC, Fodor E, Firth AE, et al. Identification of a novel splice variant form of the influenza a virus M2 ion channel with an antigenically distinct ectodomain. PLoS Pathog. 2012;8(11):e1002998.

14. Alexander DJ. A review of avian influenza in different bird species. Vet Microbiol. 2000;74(1-2):3-13.

15. Katoh K, Standley DM. MAFFT multiple sequence alignment software version 7: improvements in performance and usability. Mol Biol Evol. 2013;30(4):772-80.

16. Rice P, Longden I, Bleasby A. EMBOSS: the European molecular biology open software suite. Trends Genet. 2000;16(6):276-7.

17. Katoh K, Toh H. PartTree: an algorithm to build an approximate tree from a large number of unaligned sequences. Bioinformatics. 2007;23(3):372-4

18. Chen W, Calvo PA, Malide D, Gibbs J, Schubert U, Bacik I, Basta S, O'Neill R, Schickli J, Palese P, et al. A novel influenza a virus mitochondrial protein that induces cell death. Nat Med. 2001;7(12):1306-12.

19. Burke DF, Smith DJ. A recommended numbering scheme for influenza a HA subtypes. PLoS One. 2014;9(11):e112302.

20. Waterman MS, Arratia R, Galas DJ. Pattern recognition in several sequences: consensus and alignment. Bull Math Biol. 1984;46(4):515-27.

21. To KK, Ng KH, Que TL, Chan JM, Tsang KY, Tsang AK, Chen H, Yuen KY. Avian influenza a H5N1 virus: a continuous threat to humans. Emerg Microbes Infect. 2012;1(9):e25.

22. Hatta M, Gao P, Halfmann P, Kawaoka Y. Molecular basis for high virulence of Hong Kong H5N1 influenza a viruses. Science. 2001;293(5536):1840-2.

23. Noronha JM, Liu M, Squires RB, Pickett BE, Hale BG, Air GM, Galloway SE, Takimoto T, Schmolke M, Hunt V, et al. Influenza virus sequence feature variant type analysis: evidence of a role for NS1 in influenza virus host range restriction. J Virol. 2012;86(10):5857-66.

24. Hulse DJ, Webster RG, Russell RJ, Perez DR. Molecular determinants within the surface proteins involved in the pathogenicity of H5N1 influenza viruses in chickens. J Virol. 2004;78(18):9954-64.

25. Gohrbandt S, Veits J, Hundt J, Bogs J, Breithaupt A, Teifke JP, Weber S, Mettenleiter TC, Stech J. Amino acids adjacent to the haemagglutinin cleavage site are relevant for virulence of avian influenza viruses of subtype H5. J Gen Virol. 2011;92(1):51-9.

26. Hiromoto Y, Saito T, Lindstrom S, Nerome K. Characterization of low virulent strains of highly pathogenic a/Hong Kong/ 156/97 (H5N1) virus in mice after passage in embryonated hens' eggs. Virology. 2000;272(2):429-37.

27. Yamada S, Suzuki Y, Suzuki T, Le MQ, Nidom CA, Sakai-Tagawa Y, Muramoto Y, Ito M, Kiso M, Horimoto T, et al. Haemagglutinin mutations responsible for the binding of H5N1 influenza a viruses to human-type receptors. Nature. 2006;444(7117):378-82.

28. Auewarakul P, Suptawiwat O, Kongchanagul A, Sangma C, Suzuki Y, Ungchusak K, Louisirirotchanakul S, Lerdsamran H, Pooruk P, Thitithanyanont A, et al. An avian influenza H5N1 virus that binds to a human-type receptor. J Virol. 2007; 81(18):9950-5.

29. Yang Z-Y, Wei C-J, Kong W-P, Wu L, Xu L, Smith DF, Nabel GJ. Immunization by avian H5 influenza Hemagglutinin mutants with altered receptor binding specificity. Science. 2007;317(5839):825-8.

30. Watanabe Y, Ibrahim MS, Ellakany HF, Kawashita N, Mizuike R, Hiramatsu H, Sriwilaijaroen N, Takagi T, Suzuki Y, Ikuta K. Acquisition of human-type receptor binding specificity by new $\mathrm{H} 5 \mathrm{~N} 1$ influenza virus sublineages during their emergence in birds in Egypt. PLoS Pathog. 2011;7(5):e1002068.

31. Bean WJ, Threlkeld SC, Webster RG. Biologic potential of amantadine-resistant influenza a virus in an avian model. J Infect Dis. 1989:159(6):1050-6.

32. Nobusawa E, Sato K. Comparison of the mutation rates of human influenza a and B viruses. J Virol. 2006;80(7):3675-8.

33. Lu C, Cai Z, Zou Y, Zhang Z, Chen W, Deng L, Du X, Wu A, Yang L, Wang D, et al. FluPhenotype-a one-stop platform for early warnings of the influenza a virus. Bioinformatics. 2020;36(10):3251-3.

34. Wu H-S, Yang J-R, Liu M-T, Yang C-H, Cheng M-C, Chang F-Y. Influenza a(H5N2) virus antibodies in humans after contact with infected poultry, Taiwan, 2012. Emerg Infect Dis. 2014;20(5):857-60.

35. Liu MD, Chan TC, Wan CH, Lin HP, Tung TH, Hu FC, King CC. Changing risk awareness and personal protection measures for low to high pathogenic avian influenza in live-poultry markets in Taiwan, 2007 to 2012. BMC Infect Dis. 2015;15:241.

\section{Publisher's Note}

Springer Nature remains neutral with regard to jurisdictional claims in published maps and institutional affiliations. 